\title{
DETERMINING THE GEOMETRICAL PARAMETERS OF EXPLOITED RAIL TRACK USING APPROXIMATING SPLINE FUNCTIONS
}

\author{
G. LENDA ${ }^{1}$
}

The process of railway track adjustment is a task which includes bringing, in geometrical terms, the actual track axis to the position ensuring safe and efficient traffic of rail vehicles. The initial calculation stage of this process is to determine approximately the limits of sections of different geometry, i.e. straight lines, arcs and transition curves. This allows to draw up a draft alignment design, which is subject to control the position relative to the current state. In practice, this type of a project rarely meets the requirements associated with the values of corrective alignments. Therefore, it becomes necessary to apply iterated correction of a solution in order to determine the final project, allowing to introduce minor corrections while maintaining the assumed parameters of the route. The degree of complexity of this process is defined by the quality of determining a preliminary draft alignment design. Delimitation of the sections for creation of ereating such a design, is usually done by using the curvature diagram (InRail v8.7 Reference Guide [1], Jamka et al [2], Strach [3]), which is, however, sensitive to the misalignment of the track and measurement errors. In their paper Lenda and Strach [4] proposed a new method for creating curvature diagram, based on approximating spline function, theoretically allowing, inter alia, to reduce vulnerability to interference factors. In this study, the method to determine a preliminary draft alignment design for the track with severe overexploitation was used, and thus in the conditions adversely affecting the accuracy of the conducted readings. The results were compared to the ones obtained using classical curvature diagram. The obtained results indicate that the method allows to increase the readability of a curvature graph, which at considerable deregulation of a track takes an irregular shape, difficult to interpret. The method also favourably affects the accuracy of determining the initial parameters of the project, reducing the entire process of calculation.

Keywords: rail track adjustment, curvature graph, spline functions

\section{INTRODUCTION}

The process of railway track adjustment is a task which includes bringing, in geometrical terms, the actual track axis to the position ensuring safe and efficient traffic of rail vehicles. The calculation part of the process is related to determining the alignment project, which defines a new track axis, as well-suited as possible to a discrete set of

1 Faculty of Mining Surveying and Environmental Engineering, AGH University of Science and Technology, Kraków, e-mail: grzenda@agh.edu.pl 
observations of the real axis. This axis should meet the geometrical requirements related to the permissible speed of train traffic on a specific route. At the same time, it should require the introduction of the smallest possible physical modifications of the real axis.

The initial stage of the computation process of rail track alignment is an approximate determination of the limits of sections of different geometry, i.e. straight lines, circular arcs, and transition curves. It allows a proper selection of the measured points representing the axis of the track, and on that basis, determining the initial parameters of the rail route (the radius length of the circular arc, the length of transition curves, the location of straight sections). The alignment design determined on that basis is then subject to position control relatively to the existing points. If the displacements, which ought to be introduced on the points to receive the design shape of the track, are at a low level, usually of several centimetres, the design is accepted as the final project. Description of the rail track alignment process can be found, inter alia, in the studies: InRail v8.7 Reference Guide [1], Koc [5], Strach [3].

In practice, the design determined on the basis of the pre-selection of the points rarely fulfills the requirements. It is dependent upon numerous factors, among which the most important are: the type of a method used to determine the limits of the sections with different geometry, the degree of track misalignment, error values for determining the coordinates, the density of conducted observations, the complexity of the track geometry (e.g. occurrence of basket arches). As a result, a preliminary draft alignment design is regarded as the starting model and the final, optimal result is achieved in the process of iterated solution correction. It involves the changing of the selected, pre-set route parameters, which takes place in conjunction with controlling the location of the design, not only with reference to the measured points of the track axis, but also to the external technical devices. This is often a complex process, requiring the operator to simultaneously coordinate many factors. For that reason, it is important to determine correctly the initial parameters of the route, which allow to simplify the iterated process of reaching an optimal design.

Assigning measured points to the relevant sections is usually made based on two types of diagrams: curvatures and angles (InRail v8.7 Reference Guide [1], Lenda [6], Strach [3]. The study by Lenda and Strach [4] proposed an algorithm involving a curvature diagram based on the continuous model of the track axis, constructed using approximating spline functions. It theoretically allows for a clearer delimitation between the sections in the case of a complex geometry of the track, as well as in the case of a greater misalignment of the track and greater values of measurement errors. As a result, the algorithm is to enable an easier and more accurate determination of the initial parameters of the project, which speeds up the operator's work and also allows to determine the final project using a lower number of iterations and shortening the entire process.

This study presents an application of the proposed algorithm to determine the geometry of a rail track with a high degree of overexploitation (misalignment and technical 
wear), so in the case for which all the methods of determining the limits of the track sections may produce worse results. The effectiveness of the algorithm was compared to the results obtained by means of the classical curvature diagram. The advantages of the proposed method were evaluated in terms of accuracy of determining a preliminary regulation project and unambiguous reading of section limits.

\section{THEORETICAL ASSUMPTIONS OF RAIL TRACK GEOMETRY RECOGNITION METHODS}

The most common method of rail track geometry recognition is a curvature diagram (InRail v8.7 Reference Guide [1], Strach [7]). The curvature at straight sections is close to zero, on the transition curves it rises, to achieve a maximum, approximately constant level at the circular arcs (Figure 1.a). In order to determine the curvature, it is necessary to replace a set of point observations with a continuous model. For this purpose, it is the easiest to use circles created on the basis of successive triples of points. Since the constant curvature of a single circle is calculated on the basis of three points, a problem of proper allocation of the circles to the observed points arises. It is solved through a combined use of double-created diagram, with the curvature being assigned to the first and last of the three points of the circle, respectively Lenda [6] (Figure1.b, color black and red, respectively). Such a solution was used during the investigations, conducted for the purpose of this study. Based on information from the combined use of the diagrams, it is also possible to make one diagram, with appropriately extended limits of the sections with constant curvature (straights and circular arcs).

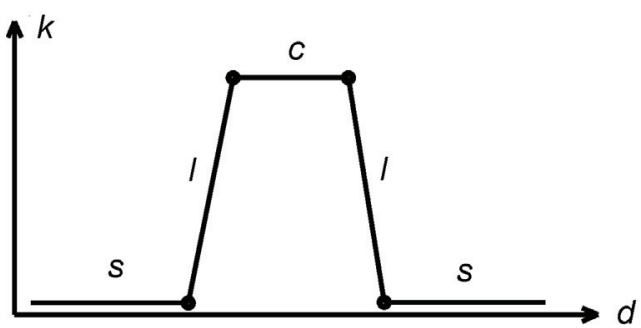

$a$

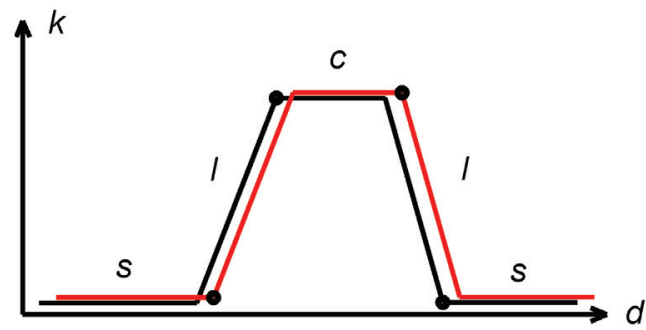

$b$

Fig. 1. a) diagram of rail track curvatures, b) diagram of the curvatures obtained from two partial diagrams (black - the curvature assigned to the first of the three points of the circle, red - to the last one). curvature $k$-curvature, $d$-mileage, $s$-straight, $l$-transition curve, $c$-circular arc Source: author's data

As a theoretical advantage of the curvature diagram are clear limits between sections of different geometry. A disadvantage is the relatively high sensitivity to misalignment of the track and the measurement errors, which adversely affects the readability of 
the diagram, from which the readings are made on the basis of visual assessment. This is especially noticeable when considering the sections measured with high density.

In order to reduce vulnerability to the above mentioned factors, in Lenda and Strach [4] a new algorithm of recognizing the geometry of rail track based on the curvature diagram was proposed. The essential change with respect to the classical curvature diagram, is a replacement of a point set of observations with approximating spline function (Diercx [8], Kiciak [9]), parameterized with a distance between the points. Its determination is achieved by minimizing the expression (2.1):

$$
r \sum_{i=1}^{n}\left(\frac{p_{i}-S\left(t_{i}\right)}{\sigma_{p_{i}}}\right)^{2}+(1-r) \int_{t_{1}}^{t_{n}}\left(S^{\prime \prime}(t)\right)^{2} d t
$$

where: $p_{i}$ - point (selected coordinate $\mathrm{x}, \mathrm{y}$ or z), $S\left(t_{i}\right)$ - value of spline function at $t_{i}, \sigma_{p i}$ - error of determining the position of a point, $r$-approximation coefficient, $t$-independent variable of function in a parametric form

Approximation spline functions allow for the damping of curve distortions, and thus of the curvature graph formed on its basis, by changing the local $\left(\sigma_{p i}\right)$, or the global $(r)$ coefficient of approximation. Assuming the same level of measurement errors $\sigma_{p i}$, smoothing the graph is best carried out using the $r \in[0,1]$ coefficient. Experimentally determined, the most advantageous values of this parameter are within the range $r \in[0.50,0.99]$, with the smaller values resulting in a greater smoothing of the graph.

A selected parameterization of the spline function (i.e. the selection of its arguments) results from the research conducted by various authors (Floater and Surazhsky [10], Haron et al [11]). They prove that in order to describe the shapes of a slowly variable curvature, and a railway track is to be considered as such, it is preferable to determine the arguments proportional to the distance between the observed points.

In order to additionally reduce the distortions in the vicinity of straight sections and arcs, a variable exponent of the curvature was used. On the curvature graph, instead of the curvature, the following value is presented:

$$
K^{e}=\left(\frac{f^{\prime \prime}(x)}{\left[1+\left(f^{\prime}(x)\right)^{2}\right]^{3 / 2}}\right)^{e}
$$

where: $K$ - curvature, $e$ - exponent

The exponents $e$ smaller than 1 damp distortions in the vicinity of the arcs, and greater than 1 damp distortions in the vicinity of the straight sections. It is preferable, therefore, to separately determine the limits of the straight sections and of the arcs, for different values of the exponent $e$. 
The algorithm, in addition to increasing the resistance of the diagram to interference factors, also allows to better to distinguish between basket arches, though it does not constitute the subject of this study.

\section{Comparison of CURVATURE Diagram PROPERTIES ON THE EXAMPLE OF OVEREXPLOITED SECTION OF RAIL TRACK}

In this comparison, two types of curvature diagrams were analyzed, as described in the previous section: classical (based on circles) and spline (based on approximating spline functions). Attention was drawn to the readability, and thus uniqueness of the conducted delimitation of the sections. Above all, however, the accuracy of determining the preliminary draft alignment design was estimated. The accuracy was compared with the final, optimal alignment design developed in the process of iterated improving of the initial solution.

The tests were carried out on the track section with considerable technical wear and misalignment. It consists of two circular arcs with associated sections of transition curves and straights (Figure 2). The track measurements were carried out for testing purposes related to the study Strach [3]. The observations were made at 5-meter intervals and thus with a considerable density, having an adverse effect on the readability of the curvature diagrams. Therefore, the relevant section has characteristics that make it potentially difficult to analyze using a curvature diagram. As a result, a preliminary draft alignment, drawn up by using it, can differ quite significantly from the optimal design.

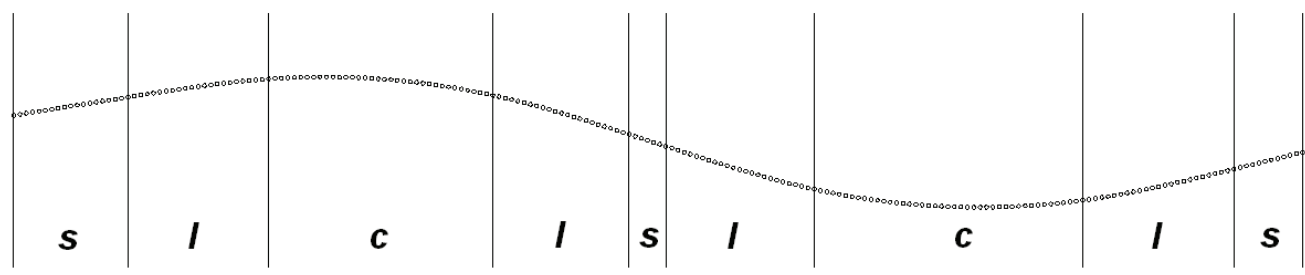

Fig.2. Analyzed track with approximate division into sections: $s$-straight line, 1 - transition curve, $c$-circular arc. Source: author's data

First, the classical curvature diagram was drawn (Figure 3). It reveals significant interferences, impeding unequivocal determination of limits. For the sections of constant curvature, a distinct fluctuation band of the diagram is visible, but it is much wider for circular arcs than straight lines. 


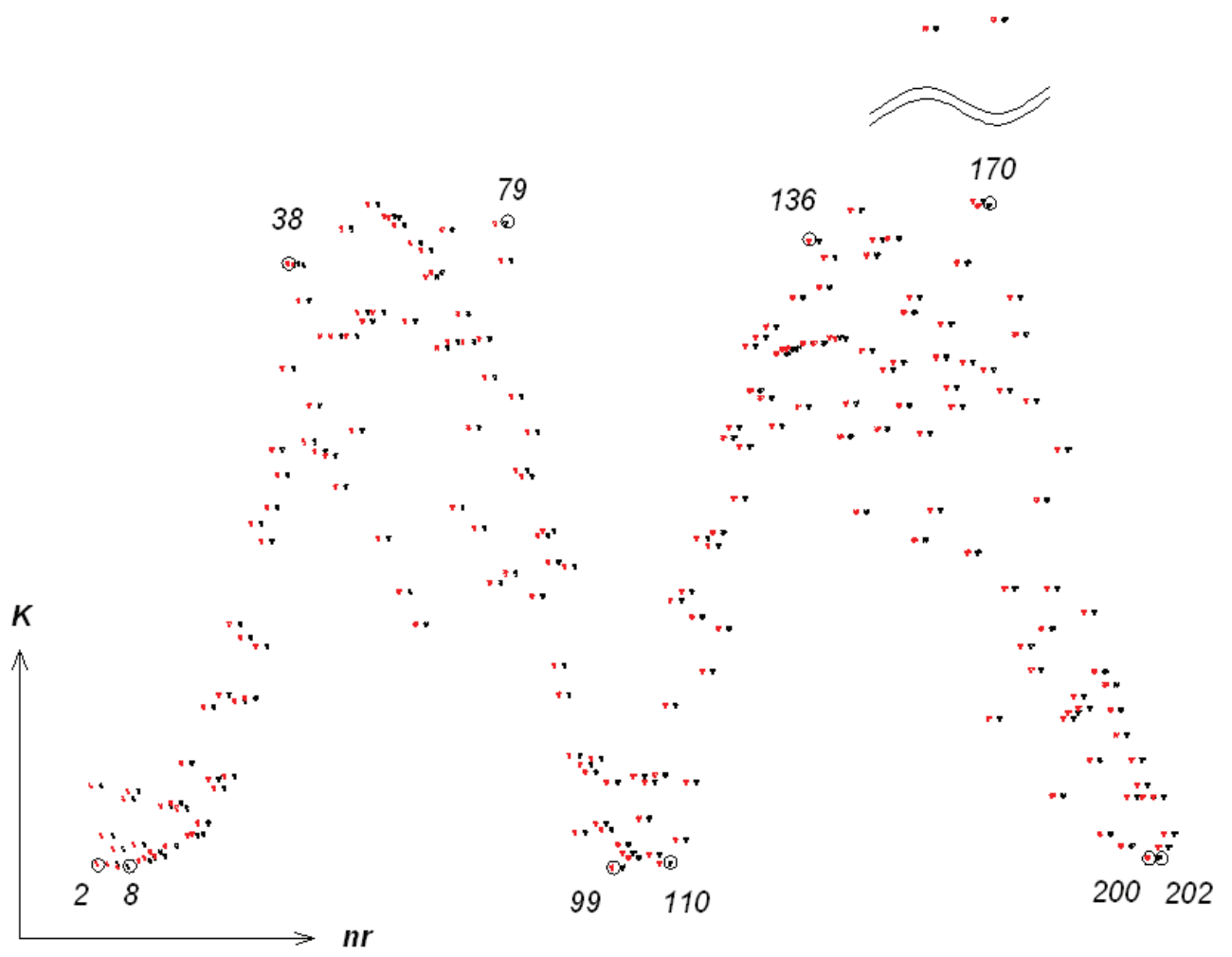

Fig.3. Classical curvature diagram (resulting from the submission of two graphs) with marked points identified as limit points of sections of different geometry. Sinuous line separates points lying significantly above the coherent diagram. Source: author's data

The end of the first section of the straight was determined in point 8 , which is located at the lowest level. Further points were also located in the fluctuation band for the straight line, but they were characterized by a steady growth of the curvature, characteristic for the points on the transition curves. Limits of the central straight based on similar observations have been fixed at points 99 and 110. The location of the third straight was set between points 200 and 202. The first circular arc having a much wider fluctuation band than the sections of the straights was determined at the widest range possible, which lies in the upper levels of the fluctuation band, setting it between points 38 and 79. Delimitation of the second arc is more questionable. A few points lying above the sinuous line had such a big curvature that its values were reduced to fit the diagram. These points were rejected from further considerations. The highest level of diagram, associated with a broader observation group was represented by points between 136 and 170. 
The curvature diagram created that was based on approximating spline functions has been presented in two Figures: 4.a and $b$. This is associated with the use of different values of spline smoothing coefficient and scalable curvature exponent, changed to recognize the section limits of straights and circular arcs. It should be noted that the classical curvature diagram shows modules of curvature on the vertical axis, and therefore the two circular arcs have been determined above the abscissa. "Spline" curvature diagram depending on the value of the curvature exponent can be either negative or positive (Figures 4.a and 4.b).
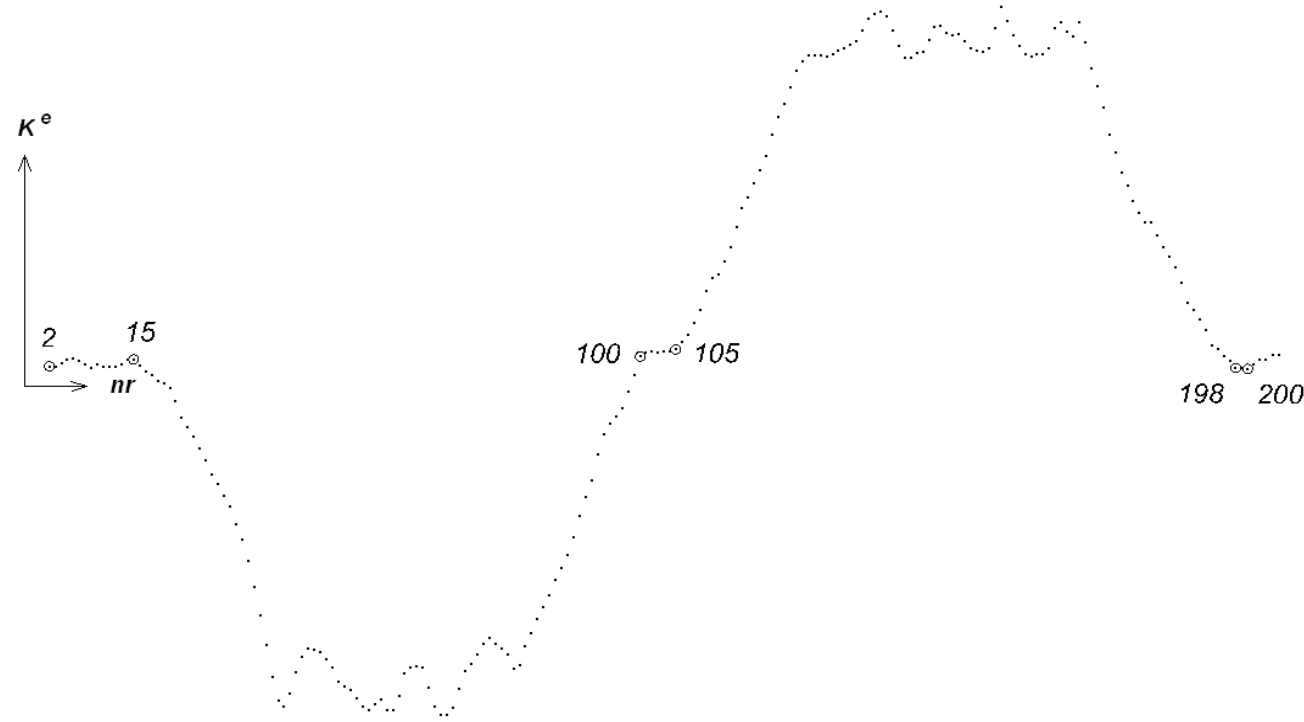

Fig.4a. "Spline" curvature diagram developed to recognize the limits of straight sections. Approximation parameter $r=0.8$, curvature exponent $e=1$

Source: author's data

The location of the straights was determined with insignificant smoothing (approximation coefficient, $r=0.8$, where $r=0$ - maximum smoothing, $r=1-$ no smoothing). Curvature exponent was left at a standard level $e=1$. With these parameters good readability of the diagrams was obtained, enough to make the readings. The end of the first straight was determined in point 15 , after which curvature change begins to be associated with a transition curve. The central section was set between points 100 and 105, which are located approximately at the same level. The last straight was located between points 198 and 200, which determined the lowest level. Each straight line was determined in the limits which are different from the ones determined with the classical curvature diagram. 


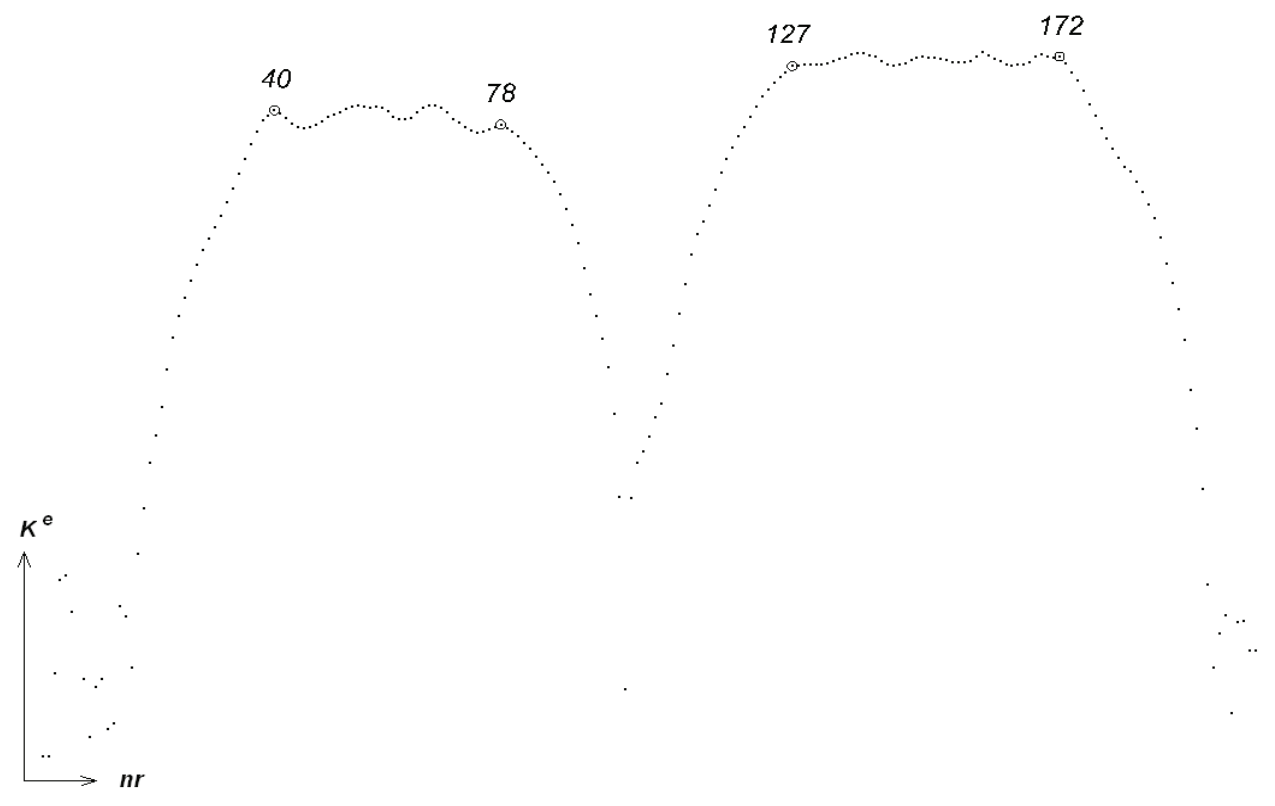

Fig.4b. "Spline" curvature diagram developed to recognize the limits of circular arcs. Approximation parameter $r=0.5$, curvature exponent $e=0.1$

Source: author's data

The location of the circular arcs was determined with greater smoothing (approximation coefficient $r=0.5$ ), due to a wider curvature fluctuation band observed for the classical diagram. The curvature exponent was decreased to the value $e=0.1$. As a result, a good suppression of the distortions around the circular arcs was obtained.

The first circular arc was established between the utmost local extremes (points 40 and 78), beyond which a systematic decrease of the curvature value begins, characteristic for the points on the transition curve. In the same way the endpoint of the second arc was determined (172). The determined starting point (127) is not in the local extreme, however it begins flattening of the course, specific to the circular arc. As for the straight sections, circular arcs limits were determined in other points than by classical curvature diagram.

In general, the clarity of the "spline" diagram is better than of a classical one, however it is necessary to check whether this is combined with a more accurate determination of the preliminary draft alignment design. For this purpose, on the basis of the identified limit points of individual sections, geometric position of the straights, radii values of the circular arcs, and the length of transition curves were determined. These data were used directly to determine the alignment designs. The model more suited to the measured points representing the existing track, thus requiring the introduction of 
smaller displacement values during alignment, should be regarded as a better one. The comparative diagram (Figure 5) presents the results for the preliminary draft alignment designs, which were based on the readings from the curvature diagrams. Diagram includes also the results for the optimal design, which was determined in the process of iterated correction of the solution. This will allows to indicate a better method of identifying section limits, as well as to evaluate its effects in relation to the desired design.

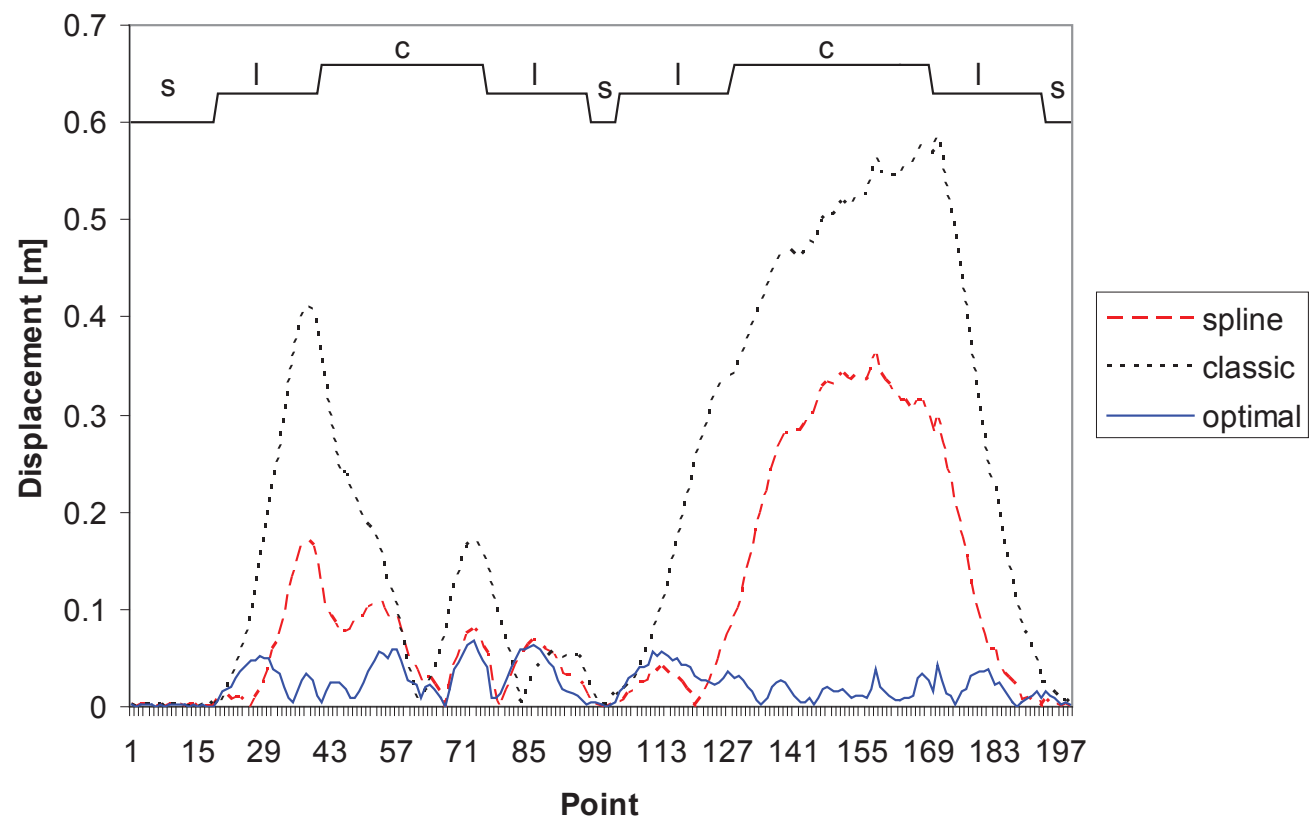

Fig.5. Displacement diagrams prepared for alignment designs created based on the data read from the curvature diagrams: classical, spline and for the optimal alignment design. The upper part of the diagram contains an approximate division into sections: $s$-straight line $l$ - transition curve, $c$-circular arc.

Source: author's data

All the projects (both draft projects and the final one) have met the minimum requirements for the values of geometrical parameters, ensuring the same classification of the route in terms of speed. As a result, the comparison of the projects regarded only the values of the alignments, determining the fitting accuracy of the preliminary regulation projects to the existing track axis.

The "spline" curvature diagram allowed for a better determination of the preliminary draft alignment design than the classical diagram. The worst results for both of the designs were obtained in the places of transition curves and circular arc connections (arc No.1) and for the entire length of the transition curves and the arc (curve No. 2), 
where they stand in a strong contrast compared to the optimal alignment design. For part of the route associated with the first circular arc (arc, transition curves, straights), the average displacement for the optimal model was $25 \mathrm{~mm}$, for the spline $-47 \mathrm{~mm}$ and for the classical $-108 \mathrm{~mm}$. "Spline" diagram of the curvatures allowed for a more than twice better determination of the design, as compared with the classical diagram. However, it is also almost twice as inaccurate as the optimal design. For a part of the route associated with the second circular arc, the average displacement for the optimal model was $21 \mathrm{~mm}, 154 \mathrm{~mm}$ for the spline one, and for the classical $-319 \mathrm{~mm}$. Therefore, a proportional distance between the initial designs created on the basis of curvature diagrams was retained, but their distance from the optimal solution increased significantly. The second circular arc showed greater misalignment and technical wear (bevel-cut rail head) as compared to the first arc, which had an adverse effect on the readings conducted using curvature diagrams. Generally, the "Spline" curvature diagram brings an overall improvement in the accuracy of the initial determination of the design, which is the basis for further modifications to obtain the optimal design.

\section{Conclusions}

The curvature diagram, formed on the basis of a track model represented by approximating spline functions, is an alternative to the classical curvature diagram, used in rail axis alignment. It demonstrates its advantages during delimitation of track sections with a high degree of overexploitation, taking into account misalignment and technical wear. It allows both to increase readability of the curvature diagram, which with a substantial misalignment of the track takes an irregular shape, and to increase the accuracy of determining a preliminary draft alignment (in the analyzed case about two times). This makes it easier and faster to determine the optimal design, which is set in the process of iterated correction of the initial solution.

\section{Acknowledgements}

This work has been made within scientific research program no. 11.11.150.005

\section{REFERENCES}

1. Bentley InRail v8.7 Reference Guide, Bentley Systems 2005

2. Jamka M., Lisowski S., Strach M.: Application of modern geodetic technologies in determining the track in the aspect of allowable train velocities. Zeszyty Naukowo - Techniczne Stowarzyszenia Inżynierów i Techników Komunikacji Rzeczpospolitej Polskiej. Oddział w Krakowie, 149, 567-581, Kraków 2009 [in Polish]

3. Strach M.: Assessement of ability of using the satelite technology RTK GPS in train track axis regulation. Ph.D. Thesis, Kraków 2003 [in Polish] 
4. Lenda G., Strach M.: Determining the railways geometry using the approximating spline functions. Pomiary Automatyka Kontrola, 12, Gliwice 2012, in print [in Polish]

5. Koc W.: Designing of the area of railway track direction in mathematical notation. Przegląd Komunikacyjny, 7-8, 96-101, 2012 [in Polish]

6. Lenda G.: Automation of the computation process of the railway track axis. Geodezja, 7, 2, 275-284, Kraków 2001 [in Polish]

7. Strach M.: Using the Bentley company's application for railway transport design._Geodezja, 12, 2, 181-189, Kraków 2006 [in Polish]

8. DieRCX P.: Curve and surface fitting with splines. Oxford University Press, New York 1995

9. KiciaK P.: Basics of modeling of curvatures and surfaces. WNT, Warszawa 2000 [in Polish]

10. Floater M. S., Surazhsky T.: Parameterization for Curve Interpolation. Studies in Computational Mathematics, 12, 39-54, 2006

11. Haron H., Rehman A., Adi D. I. S., Saba T., Lim S. P.: Parameterization Method On B-Spline Curve. Mathematical Problems in Engineering, 2012 